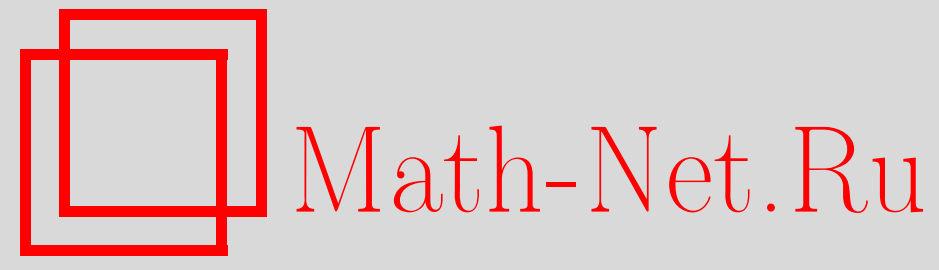

В. А. Ватутин, Структура разложимых редуцированных ветвящихся процессов. II. Функциональные предельные теоремы, Теория вероятн. и ее примен., 2015, том 60, выпуск 1, 25-44

DOI: https://doi.org/10.4213/tvp4604

Использование Общероссийского математического портала Math-Net.Ru подразумевает, что вы прочитали и согласны с пользовательским соглашением http://www . mathnet.ru/rus/agreement

Параметры загрузки:

IP : 3.85 .5 .30

26 апреля 2023 г., 16:15:17

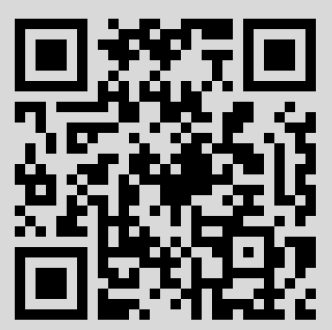




\section{СТРУКТУРА РАЗЛОЖИМЫХ РЕДУЦИРОВАННЫХ ВЕТВЯЩИХСЯ ПРОЦЕССОВ. II. ФУНКЦИОНАЛЬНЫЕ ПРЕДЕЛЬНЫЕ ТЕОРЕМЫ ${ }^{1)}$}

Рассматривается строго критический разложимый ветвящийся процесс Гальтона-Ватсона с $N$ типами частиц, занумерованными символами $1,2, \ldots, N$, в котором частицы типа $i$ могут производить потомков лишь типов $j \geqslant i$. Доказаны функциональные предельные теоремы, описывающие структуру редуцированного процесса, порожденного исходным процессом, а также найдены распределения момента рождения ближайшего общего предка всех частиц, существующих в популяции в далекий момент $n$, и типа частицы, являющейся ближайшим общим предком.

Ключевые слова и фразы: редуцированные процессы, расстояние до ближайшего общего предка, условные функциональные предельные теоремы.

1. Введение и основные результаты. В данной работе, дополняющей статью [6], мы будем рассматривать ветвящийся процесс Гальтона-Ватсона с $N$ типами частиц, занумерованными числами $1,2, \ldots, N$. Обозначим $\eta_{i, j}$ число непосредственных потомков типа $j$, произведенных частицей типа $i$ в момент ее гибели. Как и в [6], мы будем иметь дело с разложимым процессом Гальтона-Ватсона и, более того, будем предполагать, что $m_{i, j}=\mathbf{E}\left[\eta_{i, j}\right]=0$, если $j<i$. Таким образом, частицы типа $i$ анализируемого процесса могут порождать лишь потомков типов $j \geqslant i$.

В такой ситуации производящая функция закона распределения числа потомков частицы типа $i$ явно зависит лишь от $N-i+1$ аргументов:

$$
h_{i}\left(s_{1}, \ldots, s_{N}\right)=\mathbf{E}\left[s_{1}^{\eta_{i, 1}} \cdots s_{N}^{\eta_{i, N}}\right]=\mathbf{E}\left[s_{i}^{\eta_{i, i}} \cdots s_{N}^{\eta_{i, N}}\right], \quad i=1,2, \ldots, N .
$$

Будем говорить, что справедлива гипотеза $\mathbf{A}$, если разложимый ветвящийся процесс Гальтона-Ватсона с $N$ типами частиц является строго критическим, т.е.

$$
m_{i, i}=\mathbf{E}\left[\eta_{i, i}\right]=1, \quad i=1,2, \ldots, N,
$$

*Математический институт им. В.А.Стеклова РАН, Москва, Россия; е-mail: vatutin@mi.ras.ru

1) Исследование выполнено за счет гранта Российского научного фонда (проект 14-50-00005). 
и, кроме того, $m_{i, j}=0$ при $j<i$,

$$
m_{i, i+1}=\mathbf{E}\left[\eta_{i, i+1}\right] \in(0, \infty), \quad i=1,2, \ldots, N-1
$$

И

$$
\mathbf{E}\left[\eta_{i, j} \eta_{i, k}\right]<\infty, \quad i=1, \ldots, N, \quad k, j=i, i+1, \ldots, N,
$$

причем

$$
b_{i}=\frac{1}{2} \mathbf{D}\left[\eta_{i, i}\right] \in(0, \infty), \quad i=1,2, \ldots, N
$$

Пусть

$$
\mathbf{Z}(n)=\left(Z_{1}(n), \ldots, Z_{N}(n)\right)
$$

- вектор, задающий число частиц различных типов в процессе в момент $n \in \mathbb{Z}_{+}=\{0,1, \ldots\}$, a

$$
\mathbf{Z}(m, n)=\left(Z_{1}(m, n), \ldots, Z_{N}(m, n)\right)
$$

- вектор, задающий число частиц различных типов в соответствующем редуцированном процессе в момент $n$, в котором величина $Z_{i}(m, n)$ 一 это число частиц типа $i$, существующих в процессе $\mathbf{Z}(\cdot)$ в момент $m<n$ и имеющих непустое потомство в момент $n$. При этом $Z_{i}(n, n)=Z_{i}(n)$.

В дальнейшем, если не оговорено иное, мы считаем, что $\mathbf{Z}(0)=$ $(1,0, \ldots, 0)$, т.е. что эволюция процесса начинается в момент $n=0$ с одной частицы типа 1.

Положим $\gamma_{0}=0, \gamma_{i}=\gamma_{i}(N)=2^{-(N-i)}, i=1,2, \ldots, N$, и пусть $\xi^{(i)}(j)$, $i=1,2, \ldots, N, j=1,2, \ldots$, - набор независимых одинаково распределенных случайных величин, для которых

$$
\mathbf{E}\left[s^{\xi^{(i)}(j)}\right]=1-\sqrt{1-s} .
$$

Используя этот набор, мы определим на полуинтервале $0 \leqslant t<1$ разложимый кусочно постоянный ветвящийся процесс $\mathbf{R}(t)=\left(R_{1}(t), \ldots, R_{N}(t)\right)$ с непрерывным временем и $N$ типами частиц, где $R_{i}(t)$ - число частиц типа $i$ в момент $t$.

Ветвящийся процесс $\mathbf{R}(t)$ начинается в момент $t=\gamma_{0}=0$ с одной частицы типа 1 , которая живет, не производя потомства, вплоть до момента $\gamma_{1}=2^{-(N-1)}$ (но не включая этот момент). Далее, если $\gamma_{i} \leqslant t<\gamma_{i+1}, i=1,2, \ldots, N-1$, то

$$
R_{k}(t)= \begin{cases}\sum_{j=1}^{R_{i}\left(\gamma_{i}-0\right)} \xi^{(i)}(j), & \text { если } k=i+1 \\ 0, & \text { если } k \neq i+1\end{cases}
$$


Таким образом, в промежутке $\gamma_{i} \leqslant t<\gamma_{i+1}$ популяция состоит только из частиц типа $i+1$. Эти частицы появились в момент $\gamma_{i}-0$ и были порождены частицами типа $i$, которые в промежутке времени $\gamma_{i-1} \leqslant t<$ $\gamma_{i}$ не производили потомков.

Для $0 \leqslant t \leqslant 1$ введем функцию

$$
g_{n}(t)=1_{\left\{0 \leqslant t<\gamma_{1}\right\}}+g_{n} 1_{\left\{\gamma_{1} \leqslant t<1\right\}},
$$

где $g_{n}$ — монотонно возрастающая последовательность такая, что

$$
\lim _{n \rightarrow \infty} g_{n}=\infty \quad \text { и } \quad \lim _{n \rightarrow \infty} n^{-\varepsilon} g_{n}=0 \quad \text { для любого } \varepsilon>0 \text {. }
$$

В работе [6] показано, что конечномерные распределения условного процесса $\left\{\left(\mathbf{Z}\left(n^{t} g_{n}(t), n\right), 0 \leqslant t<1\right) \mid \mathbf{Z}(n) \neq \mathbf{0}\right\}$ сходятся при $n \rightarrow \infty$ к конечномерным распределениям процесса $\{\mathbf{R}(t), 0 \leqslant t<1\}$. Здесь и далее мы условимся понимать $\mathbf{Z}(x, n)$ как $\mathbf{Z}([x], n)$, где $[x]$ - целая часть числа $x$.

В данной работе мы усилим указанный результат и докажем следующее утверждение.

Теорема 1. Если справедлива гипотеза А, то для любого $i=$ $0,1,2, \ldots, N-1$ nрu $n \rightarrow \infty$

$$
\left\{\left(\mathbf{Z}\left(n^{t} g_{n}(t), n\right), \gamma_{i} \leqslant t<\gamma_{i+1}\right) \mid \mathbf{Z}(n) \neq \mathbf{0}\right\} \Longrightarrow\left\{\mathbf{R}(t), \gamma_{i} \leqslant t<\gamma_{i+1}\right\},
$$

где символ $\Longrightarrow$ обозначает сходимость в пространстве $D_{[a, b)}\left(\mathbb{Z}_{+}^{N}\right)$ функиий $\mathbf{x}(t), a \leqslant t<b$, без разрьвов второго рода со значениями $в$ пространстве $\mathbb{Z}_{+}^{N}$, оснащенном топологией Скорохода.

Нестрого говоря, теорема 1 показывает, что в условном редуцированном процессе $\left\{\mathbf{Z}\left(n^{t} g_{n}(t), n\right) \mid \mathbf{Z}(n) \neq \mathbf{0}\right\}$ фазовый переход от типа $i$ к типу $i+1$ происходит в момент $n^{\gamma_{i}}$. Природа этого фазового перехода детализирована в приводимой ниже теореме 2 , формулировка которой требует дополнительных обозначений.

Для $1 \leqslant i \leqslant N-1$ положим

$$
c_{i, N}=\left(\frac{1}{b_{N}}\right)^{1 / 2^{N-i}} \prod_{j=i}^{N-1}\left(\frac{m_{j, j+1}}{b_{j}}\right)^{1 / 2^{j-i+1}} \text { и } c_{N, N}=\frac{1}{b_{N}} .
$$

Как и в [6], введем набор

$$
\begin{aligned}
& \mathbf{U}_{i}(y)=\left(U_{i, 1}(y), \ldots, U_{i, N}(y)\right), \quad 0 \leqslant y<\infty, \quad i=1,2, \ldots, N-1, \\
& \mathbf{U}_{N}(x)=\left(U_{N, 1}(x), \ldots, U_{N, N}(x)\right), \quad 0 \leqslant x<1 \text {, }
\end{aligned}
$$

марковских процессов с непрерывным временем. Если $1 \leqslant i \leqslant N-1$, то $U_{i, j}(y) \equiv 0,0 \leqslant y<\infty, j \neq i, i+1$, в то время как пара

$$
\left(U_{i, i}(y), U_{i, i+1}(y)\right), \quad 0 \leqslant y<\infty
$$


образует однородный марковский ветвящийся процесс с непрерывным временем и частицами типов $i$ и $i+1$. Этот процесс с двумя типами частиц начинается в момент времени $y=0$ со случайного числа $U_{i, i}(0)=$ $R_{i}$ частиц типа $i$, причем

$$
\mathbf{E}\left[s_{i}^{R_{i}}\right]=\mathbf{E}\left[s_{i}^{U_{i, i}(0)}\right]=1-\left(1-s_{i}\right)^{1 / 2^{i-1}}
$$

(в частности, $U_{1,1}(0)=1$ с вероятностью 1 ). Распределение длительности жизни частиц типа $i$ является экспоненциальным с параметром $2 b_{i} c_{i, N}$. В момент гибели частица типа $i$ порождает (независимо от поведения других частиц и предыстории этого процесса) либо две частицы своего типа, либо одну частицу типа $i+1$ (каждое событие происходит с вероятностью $1 / 2)$. Частицы типа $i+1$ процесса $\mathbf{U}_{i}(\cdot)$ бессмертны и не дают потомства.

В $N$-мерном процессе $\mathbf{U}_{N}(x), 0 \leqslant x<1$, все компоненты, кроме последней, равны нулю, а компонента $U_{N, N}(\cdot)$ является неоднородным по времени марковским ветвящимся процессом с одним типом частиц. Этот процесс стартует в момент времени $x=0$ со случайного числа $U_{N, N}(0)=R_{N}$ частиц типа $N$, где

$$
\mathbf{E}\left[s_{N}^{R_{N}}\right]=\mathbf{E}\left[s_{N}^{U_{N, N}(0)}\right]=1-\left(1-s_{N}\right)^{1 / 2^{N-1}} .
$$

Продолжительность жизни каждой из $R_{N}$ первоначальных частиц типа $N$ имеет равномерное распределение на отрезке $[0,1]$. В момент гибели каждая частица порождает ровно двух потомков типа $N$ и ничего более. Если $x$ - момент гибели частицы-родителя, то продолжительность жизни каждого ее потомка имеет равномерное распределение на отрезке $[x, 1]$ (независимо от поведения других частиц и предыстории этого процесса). В момент гибели каждая частица процесса порождает ровно две частицы типа $N$ и т.д.

$\mathrm{B}$ работе [6] показано, что если справедлива гипотеза $\mathrm{A}$, а $l_{n}-$ монотонно убывающая последовательность такая, что

$$
\lim _{n \rightarrow \infty} l_{n}=0 \quad \text { и } \quad \lim _{n \rightarrow \infty} n^{\varepsilon} l_{n}=\infty \quad \text { для любого } \varepsilon>0,
$$

то при $n \rightarrow \infty$

1) для каждого $i=1, \ldots, N-1$ конечномерные распределения условного процесса

$$
\left\{\left(\mathbf{Z}\left(\left(y+l_{n}\right) n^{\gamma_{i}}, n\right), 0 \leqslant y<\infty\right) \mid \mathbf{Z}(n) \neq \mathbf{0}\right\}
$$

сходятся к конечномерным распределениям процесса $\left\{\mathbf{U}_{i}(y), 0 \leqslant y<\infty\right\}$;

2) конечномерные распределения условного процесса

$$
\left\{\left(\mathbf{Z}\left(\left(x+l_{n}\right) n, n\right), 0 \leqslant x<1\right) \mid \mathbf{Z}(n) \neq \mathbf{0}\right\}
$$

сходятся к конечномерным распределениям процесса $\left\{\mathbf{U}_{N}(x), 0 \leqslant x<1\right\}$.

В следующей теореме мы уточняем упомянутые утверждения. 
Теорема 2. Если справедлива гипотеза А, то при $n \rightarrow \infty$ 1) для каждого $i=1,2, \ldots, N-1$

$$
\left\{\left(\mathbf{Z}\left(\left(y+l_{n}\right) n^{\gamma_{i}}, n\right), 0 \leqslant y<\infty\right) \mid \mathbf{Z}(n) \neq \mathbf{0}\right\} \Longrightarrow\left\{\mathbf{U}_{i}(y), 0 \leqslant y<\infty\right\}
$$

2) $\left\{\left(\mathbf{Z}\left(\left(x+l_{n}\right) n, n\right), 0 \leqslant x<1\right) \mid \mathbf{Z}(n) \neq \mathbf{0}\right\} \Longrightarrow\left\{\mathbf{U}_{N}(x), 0 \leqslant x<1\right\}$.

Важной характеристикой редуцированного процесса является момент $\beta_{n}$ рождения ближайшего общего предка всех частиц, присутствующих в популяции в момент $n$, определяемый равенством

$$
\beta_{n}=\max \left\{m \leqslant n-1: Z_{1}(m, n)+Z_{2}(m, n)+\cdots+Z_{N}(m, n)=1\right\} .
$$

Основываясь на результатах теорем 1 и 2, мы даем в следующей теореме ответ на еще один важный вопрос: каково асимптотическое распределение момента рождения ближайшего общего предка в популяции, не вымершей к далекому моменту $n$ ?

Теорема 3. Пусть справедлива гипотеза А. Тогда

1) имеет место соотношение

$$
\lim _{n \rightarrow \infty} \mathbf{P}\left(\beta_{n} \ll n^{\gamma_{1}} \mid \mathbf{Z}(n) \neq \mathbf{0}\right)=0
$$

2) если $y \in(0, \infty)$, то для $i=1,2, \ldots, N-1$

$$
\lim _{n \rightarrow \infty} \mathbf{P}\left(\beta_{n} \leqslant y n^{\gamma_{i}} \mid \mathbf{Z}(n) \neq \mathbf{0}\right)=1-\frac{1}{2^{i}}-\frac{1}{2^{i}} e^{-2 b_{i} c_{i, N} y}
$$

3) $\partial л я i=1,2, \ldots, N-1$

$$
\lim _{n \rightarrow \infty} \mathbf{P}\left(\beta_{n} \ll n^{\gamma_{i}} \mid \mathbf{Z}(n) \neq \mathbf{0}\right)=1-\frac{1}{2^{i-1}} ;
$$

3а) $\partial л я ~ i=1,2, \ldots, N-1$

$$
\lim _{n \rightarrow \infty} \mathbf{P}\left(n^{\gamma_{i}} \ll \beta_{n} \ll n^{\gamma_{i+1}} \mid \mathbf{Z}(n) \neq \mathbf{0}\right)=0 ;
$$

4) для любого $x \in(0,1]$

$$
\lim _{n \rightarrow \infty} \mathbf{P}\left(\beta_{n} \leqslant x n \mid \mathbf{Z}(n) \neq \mathbf{0}\right)=1-\frac{1}{2^{N-1}}(1-x) .
$$

3 а м е ч а н и е. Как видно из (10), существуют промежутки времени возрастающих порядков, в каждом из которых вероятность найти ближайшего общего предка популяции, выжившей до момента $n \rightarrow \infty$, пренебрежимо мала по сравнению с вероятностью невырождения популяции к этому моменту. Более того, эти временнь́е промежутки отделены друг от друга временны́ми интервалами возрастающих порядков, внутри каждого из которых вероятность найти ближайшего общего 
предка положительна. Такое явление не имеет аналогов для неразложимых ветвящихся процессов Гальтона-Ватсона.

Наряду с распределением момента рождения ближайшего общего предка, нас будет интересовать тип $\zeta_{n}$ ближайшего общего предка популяции, не выродившейся к моменту $n$. Распределение этой случайной величины описано в следующей теореме.

Теорема 4. Пусть справедлива гипотеза А. Тогда для $i=$ $1,2, \ldots, N$

$$
p_{i}=\lim _{n \rightarrow \infty} \mathbf{P}\left(\zeta_{n}=i \mid \mathbf{Z}(n) \neq \mathbf{0}\right)=\frac{1}{2^{i}}\left(1-\delta_{i, N}\right)+\frac{1}{2^{N-1}} \delta_{i, N},
$$

где $\delta_{i, j}$ - символ Кронекера.

Заметим, что $p_{N-1}=p_{N}$.

Структура основной части статьи выглядит следующим образом. Раздел 2 содержит формулировки некоторых известных результатов, описание ряда свойств предельных процессов и формулировки необходимых утверждений из работы [6] о сходимости конечномерных распределений допредельных процессов к предельным. Раздел 3 содержит доказательства теорем 1 и 2. Наконец, раздел 4 посвящен доказательству теорем 3 и 4 .

2. Вспомогательные результаты. Для вектора $\mathbf{s}=\left(s_{1}, \ldots, s_{p}\right) \in$ $[0,1]^{p}$ (размерность $p$ которого будет обычно ясна из контекста) и вектора $\mathbf{k}=\left(k_{1}, \ldots, k_{p}\right)$ с целыми неотрицательными координатами положим

$$
\mathbf{s}^{\mathbf{k}}=s_{1}^{k_{1}} \cdots s_{p}^{k_{p}} .
$$

Пусть $\mathbf{e}_{i}-N$-мерный вектор, $i$-я компонента которого равна единице, а остальные равны нулю, а $\mathbf{1}^{(i)}$ - вектор размерности $i$, все компоненты которого равны единице.

Введем вероятностную производящую функцию

$$
H_{n}^{(i, N)}(\mathbf{s})=\mathbf{E}\left[\mathbf{s}^{\mathbf{Z}(n)} \mid \mathbf{Z}(0)=\mathbf{e}_{i}\right]=\mathbf{E}\left[s_{i}^{Z_{i}(n)} \cdots s_{N}^{Z_{N}(n)} \mid \mathbf{Z}(0)=\mathbf{e}_{i}\right]
$$

процесса $\mathbf{Z}(n)$ при условии, что этот процесс начался в нулевой момент времени с одной частицы типа $i$, и пусть

$$
\begin{gathered}
Q_{n}^{(i, N)}(\mathbf{s})=1-H_{n}^{(i, N)}(\mathbf{s}), \quad Q_{n}^{(i, N)}=1-H_{n}^{(i, N)}(\mathbf{0}), \\
m_{i, j}(n)=\mathbf{E}\left[Z_{j}(n) \mid \mathbf{Z}(0)=\mathbf{e}_{i}\right], \\
b_{j, k}(n)=\mathbf{E}\left[Z_{j}(n)\left(Z_{k}(n)-\delta_{j, k}\right) \mid \mathbf{Z}(0)=\mathbf{e}_{j}\right] .
\end{gathered}
$$

В дальнейших рассуждениях важную роль играет следующее утверждение. 
Теорема 5. Пусть $\mathbf{Z}(n), n=0,1, \ldots$, - строго критический разложимый ветвящийся процесс, удовлетворяющий гипотезе А. Тогда $m_{j, j}(n)=1$ u npu $n \rightarrow \infty$

$$
m_{i, j}(n) \sim a_{i, j} n^{j-i}, \quad i<j ; \quad b_{j, k}(n) \sim \widehat{a}_{j, k} n^{k-j+1}, \quad j \leqslant k,
$$

где $a_{i, j}$ и $\widehat{a}_{j, k}$ - положительнье константьл, явньии вид которых известен (см. [5, теорема 1]).

Кроме того (см. [4, теорема 1]), при $n \rightarrow \infty$

$$
Q_{n}^{(i, N)}=1-H_{n}^{(i, N)}(\mathbf{0})=\mathbf{P}\left(\mathbf{Z}(n) \neq \mathbf{0} \mid \mathbf{Z}(0)=\mathbf{e}_{i}\right) \sim c_{i, N} n^{-1 / 2^{N-i}},
$$

где константы $c_{i, N}$ те же, что и в (6).

Положим $\mathbf{Z}(0)=\mathbf{e}_{1}$ и обозначим

$$
T_{i}=\min \left\{n \geqslant 1: Z_{1}(n)+Z_{2}(n)+\cdots+Z_{i}(n)=0\right\}
$$

момент вырождения популяции, порожденной частицами первых $i$ типов. Согласно (12),

$$
Q_{n}^{(1, i)}=\mathbf{P}\left(T_{i}>n\right) \sim c_{1, i} n^{-2^{-(i-1)}} .
$$

Ниже нам понадобятся некоторые свойства процессов $\mathbf{R}(\cdot)$ и $\mathbf{U}_{i}(\cdot)$, $i=1,2, \ldots, N$, установленные в работе [6].

Из определения процесса $\mathbf{R}(t)$ следует, что если

$$
\gamma_{i} \leqslant t_{1}<\cdots<t_{p}<\gamma_{i+1}
$$

для некоторого $i \in\{0,1, \ldots, N-1\}$, a $\mathbf{S}_{l}=\left(s_{l, 1}, \ldots, s_{l, N}\right) \in[0,1]^{N}$, то

$$
\mathbf{E}\left[\prod_{l=1}^{p} \mathbf{S}_{l}^{\mathbf{R}\left(t_{j}\right)}\right]=1-\left(1-\prod_{l=1}^{p} s_{l, i+1}\right)^{1 / 2^{i}} .
$$

Положим

$$
\bar{X}_{R_{i}}(y ; \mathbf{s})=\mathbf{E}_{R_{i}}\left[\mathbf{s}^{\mathbf{U}_{i}(y)}\right]=\mathbf{E}_{R_{i}}\left[s_{i}^{U_{i, i}(y)} s_{i+1}^{U_{i, i+1}(y)}\right]=\bar{X}_{R_{i}}\left(y ; s_{i}, s_{i+1}\right),
$$

где выражение $\mathbf{E}_{R_{i}}[\cdot]$ обозначает, что процесс стартовал со случайного числа частиц $R_{i}$ типа $i$ с распределением (7). Как отмечено в [6],

$$
\bar{X}_{R_{i}}\left(y ; s_{i}, s_{i+1}\right)=1-\left(\varphi_{i}\left(y ; s_{i}, s_{i+1}\right)\right)^{1 / 2^{i-1}},
$$

где

$$
\varphi_{i}\left(y ; s_{i}, s_{i+1}\right)=\sqrt{1-s_{i+1}} \frac{\left(1-s_{i}\right)+\sqrt{1-s_{i+1}} \tanh \left(b_{i} c_{i, N} y \sqrt{1-s_{i+1}}\right)}{\left(1-s_{i}\right) \tanh \left(b_{i} c_{i, N} y \sqrt{1-s_{i+1}}\right)+\sqrt{1-s_{i+1}}}
$$


при $\left(s_{i}, s_{i+1}\right) \in[0,1]^{2}$, причем $\varphi_{i}(y ; 1,1)=0$ и

$$
\varphi_{i}\left(y ; s_{i}, 1\right)=\frac{1-s_{i}}{b_{i} c_{i, N} y\left(1-s_{i}\right)+1} .
$$

Пусть

$$
\bar{G}_{R_{N}}(x ; s)=\mathbf{E}_{R_{N}}\left[s^{U_{N, N}(x)}\right] .
$$

Оказывается, что (см. [6])

$$
\bar{G}_{R_{N}}(x ; s)=1-(\psi(x ; s))^{1 / 2^{N-1}},
$$

где

$$
\psi(x ; s)=\frac{1}{x+(1-x) /(1-s)}, \quad s \in[0,1], \quad x \in[0,1] .
$$

При доказательстве теорем 1 и 2 будет использован ряд утверждений, установленных в работе [6], которые нам будет удобно собрать вместе. Всюду при этом предполагается, что справедлива гипотеза А.

Пусть $\mathbf{s}=\left(s_{1}, \ldots, s_{N}\right)$, a $\mathbf{S}_{l}=\left(s_{l, 1}, \ldots, s_{l, N}\right), l=1,2, \ldots, p$.

1) Если $m \ll n^{\gamma_{i}}, i=1,2, \ldots, N$, то

$$
\lim _{n \rightarrow \infty} \mathbf{P}\left(\mathbf{Z}(m, n)=\mathbf{e}_{i} \mid \mathbf{Z}(n) \neq \mathbf{0}, \mathbf{Z}(0)=\mathbf{e}_{i}\right)=1
$$

2) Если для некоторого $i \in\{1,2, \ldots, N-1\}$

$$
n^{\gamma_{i}} \ll m_{l} \ll n^{\gamma_{i+1}}, \quad l=1,2, \ldots, p,
$$

TO

$$
\lim _{n \rightarrow \infty} \mathbf{E}\left[\prod_{l=1}^{p} \mathbf{S}_{l}^{\mathbf{Z}\left(m_{l}, n\right)} \mid \mathbf{Z}(n) \neq 0\right]=1-\left(1-\prod_{l=1}^{p} s_{l, i+1}\right)^{1 / 2^{i}} .
$$

3) Если $m \sim\left(y+l_{n}\right) n^{\gamma_{i}}, y \in[0, \infty)$, для некоторого $i \in\{1,2, \ldots, N-$ $1\}$, то

$$
\lim _{n \rightarrow \infty} \mathbf{E}\left[\mathbf{s}^{\mathbf{Z}(m, n)} \mid \mathbf{Z}(n) \neq \mathbf{0}, \mathbf{Z}(0)=\mathbf{e}_{1}\right]=1-\left(\varphi_{i}\left(y ; s_{i}, s_{i+1}\right)\right)^{1 / 2^{i-1}}
$$

4) Если $m \sim\left(x+l_{n}\right) n, x \in[0,1)$, то

$$
\lim _{n \rightarrow \infty} \mathbf{E}\left[\mathbf{s}^{\mathbf{Z}(m, n)} \mid \mathbf{Z}(n) \neq \mathbf{0}, \mathbf{Z}(0)=\mathbf{e}_{1}\right]=1-\left(\psi\left(x ; s_{N}\right)\right)^{1 / 2^{N-1}} .
$$

Пусть

$$
\begin{aligned}
J_{m, n}^{(k, N)}(\mathbf{s}) & =\mathbf{E}\left[\mathbf{s}^{\mathbf{Z}(m, n)} \mid \mathbf{Z}(n) \neq \mathbf{0}, \mathbf{Z}(0)=\mathbf{e}_{k}\right] \\
\mathbf{J}_{m, n}(\mathbf{s}) & =\left(J_{m, n}^{(1, N)}(\mathbf{s}), \ldots, J_{m, n}^{(N, N)}(\mathbf{s})\right) .
\end{aligned}
$$


Для $0 \leqslant m_{0}<m_{1}<\cdots<m_{p} \leqslant n$ положим $\mathbf{m}=\left(m_{0}, m_{1}, \ldots, m_{p}\right)$ и обозначим $\Delta_{i}=m_{i}-m_{i-1}$. Пусть далее

$$
\begin{aligned}
\widehat{J}_{m_{0}, m_{1}, \ldots, m_{p}, n}^{(i, N)}\left(\mathbf{S}_{1}, \ldots, \mathbf{S}_{p}\right) & =\widehat{J}_{\mathbf{m}, n}^{(i, N)}\left(\mathbf{S}_{1}, \ldots, \mathbf{S}_{p}\right) \\
& =\mathbf{E}\left[\prod_{l=1}^{p} \mathbf{S}_{l}^{\mathbf{Z}\left(m_{l}, n\right)} \mid \mathbf{Z}\left(m_{0}, n\right)=\mathbf{e}_{i}\right]
\end{aligned}
$$

и

$$
\widehat{\mathbf{J}}_{\mathbf{m}, n}\left(\mathbf{S}_{1}, \ldots, \mathbf{S}_{p}\right)=\left(\widehat{J}_{\mathbf{m}, n}^{(1, N)}\left(\mathbf{S}_{1}, \ldots, \mathbf{S}_{p}\right), \ldots, \widehat{J}_{\mathbf{m}, n}^{(N, N)}\left(\mathbf{S}_{1}, \ldots, \mathbf{S}_{p}\right)\right)
$$

Для $\mathbf{x}=\left(x_{1}, \ldots, x_{N}\right)$ и $\mathbf{y}=\left(y_{1}, \ldots, y_{N}\right)$ положим $\mathbf{x} \otimes \mathbf{y}=\left(x_{1} y_{1}, x_{2} y_{2}, \ldots\right.$, $\left.x_{N} y_{N}\right)$.

Следующая лемма является очевидным уточнением следствия 2 работы [7].

Лемма 1. Для любого набора $0 \leqslant m_{0}<m_{1}<\cdots<m_{p} \leqslant n$ имеем

$$
\begin{aligned}
& \widehat{J}_{\mathbf{m}, n}^{(1, N)}\left(\mathbf{S}_{1}, \ldots, \mathbf{S}_{p}\right)=\widehat{J}_{m_{0}, m_{1}, n}^{(1, N)}\left(\mathbf{S}_{1} \otimes \widehat{\mathbf{J}}_{m_{1}, m_{2}, \ldots, m_{p}, n}\left(\mathbf{S}_{2}, \ldots, \mathbf{S}_{p}\right)\right) \\
& \quad=J_{\Delta_{1}, n-m_{0}}^{(1, N)}\left(\mathbf{S}_{1} \otimes \mathbf{J}_{\Delta_{2}, n-m_{1}}\left(\mathbf{S}_{2} \otimes \cdots\left(\mathbf{S}_{p-1} \otimes \mathbf{J}_{\Delta_{p}, n-m_{p-1}}\left(\mathbf{S}_{p}\right)\right) \ldots\right)\right) .
\end{aligned}
$$

В частности, если $\mathbf{m}=\left(0, m_{1}, m_{2}\right)$, то

$$
\widehat{J}_{\mathbf{m}, n}^{(1, N)}\left(\mathbf{S}_{1}, \mathbf{S}_{2}\right)=J_{m_{1}, n}^{(1, N)}\left(\mathbf{S}_{1} \otimes \mathbf{J}_{\Delta_{2}, n-m_{1}}\left(\mathbf{S}_{2}\right)\right),
$$

a если $\mathbf{m}=\left(m_{0}, m_{1}\right)$, mo

$$
\widehat{J}_{m_{0}, m_{1}, n}^{(j, N)}(\mathbf{s})=J_{\Delta_{1}, n-m_{0}}^{(j, N)}(\mathbf{s})=1-\frac{1-H_{\Delta_{1}}^{(j, N)}\left(\mathbf{1}-(\mathbf{1}-\mathbf{s}) \otimes \mathbf{Q}_{n-m_{1}}\right)}{Q_{n-m_{0}}^{(j, N)}} .
$$

Лемма 2. Если $m_{0}=\left(Y_{0}+l_{n}\right) n^{\gamma_{i}}<m_{1}=\left(Y_{1}+l_{n}\right) n^{\gamma_{i}}$, то для любого $j \geqslant i$ существует константа $\chi \in(0, \infty)$ такая, что для всех $n \geqslant n_{0}$

$$
\mathbf{P}\left(\mathbf{Z}\left(m_{1}, n\right)=\mathbf{e}_{j} \mid \mathbf{Z}\left(m_{0}, n\right)=\mathbf{e}_{j}\right) \geqslant 1-\chi\left(Y_{1}-Y_{0}\right) .
$$

Д о к а з а т е л ь с т в о. В силу гипотезы разложимости и условия $m_{j, j}=1$, влекущего за собой равенство

$$
m_{j, j}\left(\Delta_{1}\right)=\left.\frac{\partial H_{\Delta_{1}}^{(j, N)}(\mathbf{s})}{\partial s_{j}}\right|_{\mathbf{s}=\mathbf{1}}=1
$$

получаем

$$
1-\left.\frac{\partial H_{\Delta_{1}}^{(j, N)}(\mathbf{s})}{\partial s_{j}}\right|_{\mathbf{s}=\mathbf{H}_{n-m_{1}}(\mathbf{0})} \leqslant \sum_{k=j}^{N} \mathbf{E}\left[Z_{j}\left(\Delta_{1}\right)\left(Z_{k}\left(\Delta_{1}\right)-\delta_{k, j}\right)\right] Q_{n-m_{1}}^{(k, N)} .
$$


Вспоминая (11) и (12) и полагая $h=Y_{1}-Y_{0}$, заключаем, что

$$
\begin{aligned}
\mathbf{E}\left[Z_{j}\left(\Delta_{1}\right) Z_{k}\left(\Delta_{1}\right)\right] Q_{n-m_{1}}^{(k, N)} & \leqslant c_{0}\left(n-m_{1}\right)^{-1 / 2^{N-k}}\left(\Delta_{1}\right)^{k-j+1} \\
& \leqslant c_{0}\left(n-m_{1}\right)^{-1 / 2^{N-k}}\left(h n^{1 / 2^{N-i}}\right)^{k-j+1} \\
& \leqslant \chi h n^{-1 / 2^{N-k}}\left(n^{1 / 2^{N-i}}\right)^{k-j+1}
\end{aligned}
$$

для некоторых констант $0<c_{0} \leqslant \chi<\infty$. Ввиду неравенств $k \geqslant j \geqslant i$ имеем

$$
\frac{k-j+1}{2^{N-i}}-\frac{1}{2^{N-k}}=\frac{1}{2^{N-i}}\left(k-j+1-2^{k-i}\right) \leqslant 0 .
$$

Таким образом,

$$
1-\left.\frac{\partial H_{\Delta_{1}}^{(j, N)}(\mathbf{s})}{\partial s_{j}}\right|_{\mathbf{s}=\mathbf{H}_{n-m_{1}}(\mathbf{0})} \leqslant \chi h .
$$

Отсюда, используя предыдущую лемму и монотонность функции $Q_{r}^{(j, N)}$ по $r$, получаем

$$
\begin{aligned}
\mathbf{P}\left(\mathbf{Z}\left(m_{1}, n\right)=\mathbf{e}_{j} \mid \mathbf{Z}\left(m_{0}, n\right)=\mathbf{e}_{j}\right) & =\frac{\left.Q_{n-m_{1}}^{(j, N)} \frac{\partial H_{\Delta_{1}}^{(j, N)}(\mathbf{s})}{\partial s_{j}}\right|_{\mathbf{s}=\mathbf{H}_{n-m_{1}}(\mathbf{0})}}{Q_{n-m_{0}}^{(j, N)}} \\
& \geqslant\left.\frac{\partial H_{\Delta_{1}}^{(j, N)}(\mathbf{s})}{\partial s_{j}}\right|_{\mathbf{s}=\mathbf{H}_{n-m_{1}}(\mathbf{0})} \geqslant 1-\chi h .
\end{aligned}
$$

Лемма 2 доказана.

3. Плотность. Обозначим через $\mathbf{z}^{(i, i+1)}$ вектор размерности $N-2$, полученный из вектора $\mathbf{z}=\left(z_{1}, \ldots, z_{N}\right) \in \mathbb{Z}_{+}^{N}$ вычеркиванием координат $i$ и $i+1$, через $\mathbf{z}^{(i)}$ вектор размерности $N-1$, полученный из вектора $\mathbf{z}$ вычеркиванием $i$-й координаты, а через $\|\mathbf{z}\|$ сумму абсолютных значений координат вектора $\mathbf{z}$.

Пусть $\mathscr{C}_{i}=\left\{\mathbf{z} \in \mathbb{Z}_{+}^{N}:\left\|\mathbf{z}^{(i)}\right\|>0\right\}, \mathscr{B}_{i}=\mathbb{Z}_{+}^{N} \backslash \mathscr{C}_{i}$ и

$$
\mathscr{C}_{i, i+1}=\left\{\mathbf{z} \in \mathbb{Z}_{+}^{N}:\left\|\mathbf{z}^{(i, i+1)}\right\|>0\right\}
$$

Положим $\underline{Z}_{i}(m)=Z_{1}(m)+\cdots+Z_{i}(m)$ и пусть

$$
\underline{Z}_{i}(m, n)=\sum_{k=1}^{i} Z_{k}(m, n), \quad \bar{Z}_{i}(m, n)=\sum_{k=i}^{N} Z_{k}(m, n) .
$$

В дальнейшем нам будет удобно использовать обозначение $\mathbf{P}_{n}(\mathscr{B})$ вместо $\mathbf{P}\left(\mathscr{B} \mid \mathbf{Z}(n) \neq \mathbf{0}, \mathbf{Z}(0)=\mathbf{e}_{1}\right)$ для любого допустимого события $\mathscr{B}$.

Мы начинаем проверку желаемой плотности допредельных процессов из теорем 1 и 2 с доказательства двух важных лемм.

Пусть $A_{i}(n)=\left\{m: n^{\gamma_{i}} g_{n}\left(\gamma_{i}\right) \leqslant m<n^{\gamma_{i+1}-\varepsilon} g_{n}\left(\gamma_{i+1}-\varepsilon\right)\right\}, \varepsilon \in\left(0, \gamma_{1}\right)$. 
Лемма 3. Для любых $i \in\{0,1,2, \ldots, N-1\} u \varepsilon \in\left(0, \gamma_{1}\right)$

$$
\lim _{n \rightarrow \infty} \mathbf{P}_{n}\left(\exists m \in A_{i}(n): \mathbf{Z}(m, n) \in \mathscr{C}_{i+1}\right)=0 .
$$

Д о к а з а т ельс т в о. Если $m \in A_{i}(n)$, то

$$
\bar{Z}_{i+2}(m, n) \leqslant \bar{Z}_{i+2}\left(n^{\gamma_{i+1}-\varepsilon} g_{n}\left(\gamma_{i+1}-\varepsilon\right), n\right)
$$

и

$$
\left\{\underline{Z}_{i}(m, n)>0\right\} \subseteq\left\{\underline{Z}_{i}(m)>0\right\} \subseteq\left\{\underline{Z}_{i}\left(n^{\gamma_{i}} g_{n}\left(\gamma_{i}\right)\right)>0\right\} .
$$

Таким образом,

$$
\begin{aligned}
\mathbf{P}_{n}\left(\exists m \in A_{i}(n): \mathbf{Z}(m, n) \in \mathscr{C}_{i+1}\right) \leqslant & \mathbf{P}_{n}\left(\bar{Z}_{i+2}\left(n^{\gamma_{i+1}-\varepsilon} g_{n}\left(\gamma_{i+1}-\varepsilon\right), n\right)>0\right) \\
& +\mathbf{P}_{n}\left(\underline{Z}_{i}\left(n^{\gamma_{i}} g_{n}\left(\gamma_{i}\right)\right)>0\right) .
\end{aligned}
$$

Устремляя $n$ к бесконечности, мы видим, что первое слагаемое в правой части этого неравенства стремится к нулю в силу (16), в то время как второе слагаемое равно нулю при $i=0$ и стремится к нулю при $1 \leqslant i \leqslant$ $N-1$ в силу оценок

$$
\begin{aligned}
\mathbf{P}_{n}\left(\mathbf{Z}_{i-1}\left(n^{\gamma_{i}} g_{n}\left(\gamma_{i}\right)\right)>0\right) & =\frac{\mathbf{P}\left(T_{i}>n^{\gamma_{i}} g_{n}\left(\gamma_{i}\right)\right)}{\mathbf{P}\left(T_{N}>n\right)} \\
& \sim \frac{c_{1, i}}{c_{1, N}} \frac{n^{1 / 2^{N-1}}}{\left(n^{1 / 2^{N-i}} g_{n}\left(\gamma_{i}\right)\right)^{1 / 2^{i-1}}}=\frac{c_{1, i}}{c_{1, N}} \frac{1}{\left(g_{n}\left(\gamma_{i}\right)\right)^{1 / 2^{i-1}}},
\end{aligned}
$$

следующих из (13). Лемма 3 доказана.

Лемма 4. Если $N \geqslant 3$, то для любого $i=1,2, \ldots, N-1$ и достаточно малого $\varepsilon>0$

$$
\lim _{n \rightarrow \infty} \mathbf{P}_{n}\left(\exists m \in\left[n^{3 \gamma_{i-1}}, n^{\gamma_{i+1}-\varepsilon}\right]: \mathbf{Z}(m, n) \in \mathscr{C}_{i, i+1}\right)=0 .
$$

Д о к а з а т е л ь с т в о. При помощи тех же соображений, что и в доказательстве леммы 3 , заключаем, что

$$
\begin{aligned}
& \mathbf{P}_{n}\left(\exists m \in\left[n^{3 \gamma_{i-1}}, n^{\gamma_{i+1}-\varepsilon}\right]: \mathbf{Z}(m, n) \in \mathscr{C}_{i, i+1}\right) \\
& \quad \leqslant \mathbf{P}_{n}\left(\bar{Z}_{i+2}\left(n^{\gamma_{i+1}-\varepsilon}, n\right)>0\right)+\mathbf{P}_{n}\left(\underline{Z}_{i-1}\left(n^{3 \gamma_{i-1}}\right)>0\right) .
\end{aligned}
$$

В силу (16) первое слагаемое стремится к нулю при $n \rightarrow \infty$, в то время как второе слагаемое при $i=1$ равно нулю по определению, а при $i \geqslant 2$ его можно оценить следующим образом:

$$
\frac{\mathbf{P}\left(T_{i-1}>n^{3 \gamma_{i-1}}\right)}{\mathbf{P}\left(T_{N}>n\right)} \sim \frac{c_{1, i-1}}{c_{1, N}} \frac{n^{1 / 2^{N-1}}}{\left(n^{3 / 2^{N-i+1}}\right)^{1 / 2^{i-2}}} \sim \frac{c_{1, i-1}}{c_{1, N}} \frac{1}{n^{1 / 2^{N-2}}} .
$$

Этим завершается доказательство леммы 4. 
3.1. Макроскопический взгляд. В этом пункте мы докажем теорему 1, которая описывает макроскопическую структуру генеалогического дерева процесса.

Сходимость конечномерных распределений условного процесса $\left\{\mathbf{Z}\left(n^{t} g_{n}(t), n\right), 0 \leqslant t<1 \mid \mathbf{Z}(n) \neq \mathbf{0}, \mathbf{Z}(0)=\mathbf{e}_{1}\right\}$ к соответствующим конечномерным распределениям процесса $\{\mathbf{R}(t), 0 \leqslant t<1\}$ была установлена в теореме 1 работы [6]. Поэтому мы сконцентрируемся на доказательстве плотности.

Поскольку процесс $\mathbf{Z}\left(n^{t} g_{n}(t), n\right)$ имеет целочисленные компоненты, нам необходимо проверить, что для каждого отрезка $A_{i}=\left[\gamma_{i}, \gamma_{i+1}-\varepsilon\right]$, $i=0,1, \ldots, N-1$, выполнено следующее (см. [1, теорема 15.3]):

1) для любого положительного числа $\eta$ существует число $L$ такое, что

$$
\mathbf{P}_{n}\left(\sup _{t \in A_{i}}\left\|\mathbf{Z}\left(n^{t} g_{n}(t), n\right)\right\|>L\right) \leqslant \eta, \quad n \geqslant 1
$$

2 ) для любого положительного числа $\eta$ существуют $\delta>0$ и $n_{0}$ такие, что для всех $n \geqslant n_{0}$

$$
\mathbf{P}_{n}\left(\max \left(\min _{k=1,2}\left\|\mathbf{Z}\left(n^{t} g_{n}(t), n\right)-\mathbf{Z}\left(n^{t_{k}} g_{n}\left(t_{k}\right), n\right)\right\|\right) \neq 0\right) \leqslant \eta
$$

(здесь максимум взят по всем $\gamma_{i} \leqslant t_{1} \leqslant t \leqslant t_{2} \leqslant \gamma_{i+1}-\varepsilon$ таким, что $\left.t_{2}-t_{1} \leqslant \delta\right)$

$$
\mathbf{P}_{n}\left(\exists t, s \in\left[\gamma_{i}, \gamma_{i}+\delta\right]: \mathbf{Z}\left(n^{t} g_{n}(t), n\right) \neq \mathbf{Z}\left(n^{s} g_{n}(s), n\right)\right) \leqslant \eta
$$

и

$$
\mathbf{P}_{n}\left(\exists t, s \in\left[\gamma_{i+1}-\delta-\varepsilon, \gamma_{i+1}-\varepsilon\right]: \mathbf{Z}\left(n^{t} g_{n}(t), n\right) \neq \mathbf{Z}\left(n^{s} g_{n}(s), n\right)\right) \leqslant \eta
$$

Монотонность случайной величины $\left\|\mathbf{Z}\left(n^{t} g_{n}(t), n\right)\right\|$ по $t$ при фиксированном $n$ существенно упрощает доказательство.

Действительно, в этом случае

$$
\mathbf{P}_{n}\left(\sup _{t \in A_{i}}\left\|\mathbf{Z}\left(n^{t} g_{n}(t), n\right)\right\|>L\right) \leqslant \mathbf{P}_{n}\left(\left\|\mathbf{Z}\left(n^{1-\varepsilon} g_{n}(1-\varepsilon), n\right)\right\|>L\right)
$$

и (21) следует из сходимости одномерных распределений, установленной в (16) для случая $p=1, i=N-1$.

Для доказательства (22)-(24) введем события

$$
\begin{aligned}
\mathscr{D}_{i} & =\left\{\forall t \in A_{i}: \mathbf{Z}\left(n^{t} g_{n}(t), n\right) \in \mathscr{B}_{i+1}\right\} \\
\mathscr{F}_{i}(a, b) & =\left\{\exists t, s \in[a, b]: Z_{i+1}\left(n^{t} g_{n}(t), n\right) \neq Z_{i+1}\left(n^{s} g_{n}(s), n\right)\right\},
\end{aligned}
$$


выберем достаточно малое $\delta>0$ и заметим, что если $[a, b] \subset\left[\gamma_{i}, \gamma_{i+1}-\varepsilon\right]$, TO

$$
\begin{aligned}
& \mathbf{P}_{n}\left(\exists t, s \in[a, b]: \mathbf{Z}\left(n^{t} g_{n}(t), n\right) \neq \mathbf{Z}\left(n^{s} g_{n}(s), n\right)\right) \\
& \quad \leqslant \mathbf{P}_{n}\left(\exists t \in A_{i}: \mathbf{Z}\left(n^{t} g_{n}(t), n\right) \in \mathscr{C}_{i+1}\right)+\mathbf{P}_{n}\left(\mathscr{D}_{i} \cap \mathscr{F}_{i}\left(\gamma_{i}, \gamma_{i+1}-\varepsilon\right)\right) .
\end{aligned}
$$

В силу леммы 3 первый член в правой части стремится к нулю при $n \rightarrow \infty$. Далее, для $i \geqslant 1$

$$
\mathbf{P}_{n}\left(\mathscr{D}_{i} \cap \mathscr{F}_{i}\left(\gamma_{i}, \gamma_{i+1}-\varepsilon\right)\right) \leqslant \mathbf{P}_{n}\left(Z_{i+1}\left(n^{\gamma_{i}} g_{n}, n\right) \neq Z_{i+1}\left(n^{\gamma_{i+1}-\varepsilon} g_{n}, n\right)\right) \rightarrow 0
$$

в силу (16). Это доказывает (23) и (24).

Для проверки справедливости соотношения (22) осталось заметить, что

$$
\begin{aligned}
\mathbf{P}_{n}\left(\max \left(\min _{k=1,2}\left\|\mathbf{Z}\left(n^{t} g_{n}(t), n\right)-\mathbf{Z}\left(n^{t_{k}} g_{n}\left(t_{k}\right), n\right)\right\|\right) \neq 0\right) \\
\leqslant \mathbf{P}_{n}\left(\exists t, s \in\left[\gamma_{i}, \gamma_{i+1}-\varepsilon\right]: \mathbf{Z}\left(n^{t} g_{n}(t), n\right) \neq \mathbf{Z}\left(n^{s} g_{n}(s), n\right)\right),
\end{aligned}
$$

и использовать такие же рассуждения, как и раньше. Теорема 1 доказана.

3.2. Микроскопический взгляд. В этом пункте мы опираемся на идеи работы [3] и в связи с этим формулируем частный и слегка модифицированный случай теоремы 6.5.4 из [2], дающий критерий сходимости марковских процессов в топологии Скорохода.

Пусть $\mathbf{K}_{n}(y), n=1,2, \ldots$, - последовательность марковских процессов со значениями в $\mathbb{Z}_{+}^{N}$, траектории которых принадлежат с вероятностью 1 пространству $D_{[a, b]}\left(\mathbb{Z}_{+}^{N}\right)$ функций без разрывов второго рода на отрезке $[a, b]$.

Теорема 6. Если конечномернье распределения процессов $\left\{\mathbf{K}_{n}(y)\right.$, $a \leqslant y \leqslant b\}$ сходятся при $n \rightarrow \infty \kappa$ соответствуюшим конечномерным распределениям прочесса $\{\mathbf{K}(y), a \leqslant y \leqslant b\}$ и существует разбиение $\mathbb{Z}_{+}^{N}=\mathscr{B} \cup \mathscr{C}, \mathscr{B} \cap \mathscr{C}=\varnothing$ maкое, ито

$$
\lim _{h \downarrow 0} \varlimsup_{n \rightarrow \infty} \sup _{0 \leqslant s-y \leqslant h} \sup _{\mathbf{z} \in \mathscr{B}} \mathbf{P}\left(\mathbf{K}_{n}(s) \neq \mathbf{K}_{n}(y) \mid \mathbf{K}_{n}(y)=\mathbf{z}\right)=0
$$

$u$

$$
\lim _{n \rightarrow \infty} \mathbf{P}\left(\exists y \in[a, b]: \mathbf{K}_{n}(y) \in \mathscr{C}\right)=0
$$

mo npu $n \rightarrow \infty$

$$
\left\{\mathbf{K}_{n}(y), a \leqslant y \leqslant b\right\} \Longrightarrow\{\mathbf{K}(y), a \leqslant y \leqslant b\}
$$


В силу леммы 1 закон распределения

$$
\mathbf{P}_{n}(\{\mathbf{Z}(m, n), 0 \leqslant m \leqslant n\} \in(\cdot) \mid \mathbf{Z}(n) \neq \mathbf{0})
$$

задает, при каждом фиксированном $n$, неоднородный марковский ветвящийся процесс. Обозначим через $\mathbf{P}_{n}\left(m_{1}, \mathbf{z} ; m_{2},(\cdot)\right)$ переходные вероятности этого процесса.

Для доказательства плотности распределений процессов

$$
\left\{\left(\mathbf{Z}\left(\left(y+l_{n}\right) n^{\gamma_{i}}, n\right), 0 \leqslant y<\infty\right) \mid \mathbf{Z}(n) \neq \mathbf{0}\right\}, \quad i=1,2, \ldots, N-1,
$$

нам необходимо построить соответствующее разбиение пространства $\mathbb{Z}_{+}^{N}$ и использовать теорему 6 при каждом $[0, b] \subset[0, \infty)$.

Заметим, что если $\mathbf{w}=\left(w_{1}, \ldots, w_{N}\right) \leqslant \mathbf{z}=\left(z_{1}, \ldots, z_{N}\right)$ (где знак неравенства понимается покомпонентно), то

$$
\mathbf{P}_{n}\left(m_{0}, \mathbf{w} ; m_{1},\{\mathbf{w}\}\right) \geqslant \mathbf{P}_{n}\left(m_{0}, \mathbf{z} ; m_{1},\{\mathbf{z}\}\right) .
$$

Положим $\mathscr{C}(k)=\left\{\mathbf{z} \in \mathbb{Z}_{+}^{N}:\|\mathbf{z}\| \leqslant k\right\}$,

$$
\mathscr{C}_{i}(k)=\left\{\mathbf{z} \in \mathbb{Z}_{+}^{N}: z_{1}+\cdots+z_{i-1}>0 ;\|\mathbf{z}\| \leqslant k\right\}, \quad \mathscr{J}_{i}(k)=\mathscr{C}(k) \backslash \mathscr{C}_{i}(k),
$$

зафиксируем $i \in\{1, \ldots, N-1\}$ и обозначим $m_{j}=\left(Y_{j}+l_{n}\right) n^{\gamma_{i}}, j=1,2$.

Лемма 5. Если справедлива гипотеза А, то для любых фиксированных $k$ и $0<b<\infty$

$$
\lim _{h \downarrow 0} \varlimsup_{n \rightarrow \infty} \sup _{\substack{0 \leqslant Y_{1}-Y_{0} \leqslant h \\ Y_{1}, Y_{0} \in[0, b]}} \sup _{\mathbf{z} \in \mathscr{J}_{i}(k)} \mathbf{P}_{n}\left(\mathbf{Z}\left(m_{1} ; n\right) \neq \mathbf{z} \mid \mathbf{Z}\left(m_{0} ; n\right)=\mathbf{z}\right)=0 .
$$

Д о к а з а т е л ь с т в о. В силу свойства ветвления, предположения о разложимости исходного процесса и положительности числа потомков у каждой частицы редуцированного процесса, имеем для всех $m_{1} \geqslant m_{0}$ и $\mathbf{z} \in \mathscr{J}_{i}(k):$

$$
\begin{aligned}
\mathbf{P}_{n}\left(m_{0}, \mathbf{z} ; m_{1},\{\mathbf{z}\}\right) & =\prod_{j=i}^{N}\left(\mathbf{P}_{n}\left(m_{0}, \mathbf{e}_{j} ; m_{1},\left\{\mathbf{e}_{j}\right\}\right)\right)^{z_{j}} \\
& \geqslant \prod_{j=i}^{N}\left(\mathbf{P}_{n}\left(m_{0}, \mathbf{e}_{j} ; m_{1},\left\{\mathbf{e}_{j}\right\}\right)\right)^{k} .
\end{aligned}
$$

Используя лемму 2 , получаем для $m_{0}=\left(Y_{0}+l_{n}\right) n^{\gamma_{i}}$ и $m_{1}=\left(Y_{1}+l_{n}\right) n^{\gamma_{i}}$ :

$$
\inf _{\substack{0 \leqslant Y_{1}-Y_{0} \leqslant h \\ Y_{0}, Y_{1} \in[0, b]}} \mathbf{P}_{n}\left(m_{0}, \mathbf{z} ; m_{1},\{\mathbf{z}\}\right) \geqslant(1-\chi h)^{N k} .
$$

Эта оценка влечет за собой утверждение леммы 5. 
Лемма 6. Если $m_{j}=\left(Y_{j}+l_{n}\right) n^{\gamma_{i}}, j=0,1,2, u 0 \leqslant Y_{0}<Y_{1}<Y_{2} c$ $Y_{1}-Y_{0} \leqslant h$, то для всех $n \geqslant n_{0}$

$$
\begin{aligned}
& \mathbf{P}_{n}\left(\mathbf{Z}\left(m_{1}, n\right)=\mathbf{z} \mid \mathbf{Z}\left(m_{0}, n\right)=\mathbf{z} ;\left\|\mathbf{Z}\left(m_{2}, n\right)\right\| \leqslant k\right) \\
& \quad \geqslant \mathbf{P}_{n}\left(m_{0}, \mathbf{z} ; m_{1},\{\mathbf{z}\}\right) \frac{\mathbf{P}_{n}\left(m_{1}, \mathbf{z} ; m_{2}, \mathscr{C}(k)\right)}{\mathbf{P}_{n}\left(m_{1}, \mathbf{z} ; m_{2}, \mathscr{C}(k)\right)+\chi N k h} .
\end{aligned}
$$

Д ок а з а те ль с т в о. Имеем

$$
\begin{gathered}
\mathbf{P}_{n}\left(\mathbf{Z}\left(m_{1}, n\right)=\mathbf{z} \mid \mathbf{Z}\left(m_{0}, n\right)=\mathbf{z},\left\|\mathbf{Z}\left(m_{2}, n\right)\right\| \leqslant k\right) \\
=\mathbf{P}_{n}\left(m_{0}, \mathbf{z} ; m_{1},\{\mathbf{z}\}\right) \frac{\mathbf{P}_{n}\left(m_{1}, \mathbf{z} ; m_{2}, \mathscr{C}(k)\right)}{\mathbf{P}_{n}\left(m_{0}, \mathbf{z} ; m_{2}, \mathscr{C}(k)\right)}
\end{gathered}
$$

В силу (25)

$$
\begin{aligned}
\mathbf{P}_{n}\left(m_{0}, \mathbf{z} ; m_{2}, \mathscr{C}(k)\right) & =\sum_{\mathbf{z}^{\prime}} \mathbf{P}_{n}\left(m_{0}, \mathbf{z} ; m_{1},\left\{\mathbf{z}^{\prime}\right\}\right) \mathbf{P}_{n}\left(m_{1}, \mathbf{z}^{\prime} ; m_{2}, \mathscr{C}(k)\right) \\
& \leqslant 1-\mathbf{P}_{n}\left(m_{0}, \mathbf{z} ; m_{1},\{\mathbf{z}\}\right)+\mathbf{P}_{n}\left(m_{1}, \mathbf{z} ; m_{2}, \mathscr{C}(k)\right) \\
& \leqslant 1-(1-\chi h)^{N k}+\mathbf{P}_{n}\left(m_{1}, \mathbf{z} ; m_{2}, \mathscr{C}(k)\right) \\
& \leqslant \chi N k h+\mathbf{P}_{n}\left(m_{1}, \mathbf{z} ; m_{2}, \mathscr{C}(k)\right) .
\end{aligned}
$$

Отсюда и вытекает необходимое утверждение.

Лемма 7. Если справедлива гипотеза А, то для любых фиксированньих $k, 0<b<\infty u$

$$
m_{0}=\left(Y_{0}+l_{n}\right) n^{\gamma_{i}}, \quad m_{1}=\left(Y_{1}+l_{n}\right) n^{\gamma_{i}}, \quad m_{2}=2 b n^{\gamma_{i}}
$$

справедливо соотношение

$$
\begin{aligned}
& \lim _{h \downarrow 0} \varlimsup_{n \rightarrow \infty} \sup _{\substack{0 \leqslant Y_{1}-Y_{0} \leqslant h \\
Y_{1}, Y_{0} \in[0, b]}} \sup _{\mathbf{z} \in \mathscr{J}_{i}(k)} \mathbf{P}_{n}\left(\mathbf{Z}\left(m_{1} ; n\right) \neq \mathbf{z} \mid \mathbf{Z}\left(m_{0} ; n\right)=\mathbf{z},\right. \\
&\left.\left\|\mathbf{Z}\left(m_{2}, n\right)\right\| \leqslant k\right)=0 .
\end{aligned}
$$

Д о к а з а т е л ь с т в о. В силу неравенства (25) и леммы 6 для $m_{0}=\left(Y_{0}+l_{n}\right) n^{\gamma_{i}}$ и $m_{1}=\left(Y_{1}+l_{n}\right) n^{\gamma_{i}}$ имеем

$$
\begin{gathered}
\mathbf{P}_{n}\left(\mathbf{Z}\left(m_{1}, n\right)=\mathbf{z} \mid \mathbf{Z}\left(m_{0}, n\right)=\mathbf{z} ;\left\|\mathbf{Z}\left(m_{2}, n\right)\right\| \leqslant k\right) \\
\geqslant(1-\chi h)^{N k} \frac{\mathbf{P}_{n}\left(m_{1}, \mathbf{z} ; m_{2}, \mathscr{C}(k)\right)}{\mathbf{P}_{n}\left(m_{1}, \mathbf{z} ; m_{2}, \mathscr{C}(k)\right)+\chi N k h} .
\end{gathered}
$$

Используя предположение о разложимости и лемму 2 , получаем

$$
\begin{aligned}
\mathbf{P}_{n}\left(m_{1}, \mathbf{z} ; m_{2}, \mathscr{C}(k)\right) \geqslant \mathbf{P}_{n}\left(m_{1}, \mathbf{z} ; m_{2},\{\mathbf{z}\}\right) \\
\quad=\prod_{j=i}^{N}\left(\mathbf{P}_{n}\left(m_{1}, \mathbf{e}_{j} ; m_{2},\left\{\mathbf{e}_{j}\right\}\right)\right)^{z_{j}} \geqslant \prod_{j=i}^{N} \mathbf{P}_{n}^{k}\left(l_{n} n^{\gamma_{i}}, \mathbf{e}_{j} ; 2 b n^{\gamma_{i}},\left\{\mathbf{e}_{j}\right\}\right) .
\end{aligned}
$$


Из (15) и (16) следует, что $\lim _{n \rightarrow \infty} \prod_{j=i}^{N} \mathbf{P}_{n}^{k}\left(l_{n} n^{\gamma_{i}}, \mathbf{e}_{j} ; 2 b n^{\gamma_{i}},\left\{\mathbf{e}_{j}\right\}\right)=\mathbf{P}^{k}\left(\mathbf{U}_{i}(2 b)=\mathbf{e}_{i} \mid \mathbf{U}_{i}(0)=\mathbf{e}_{i}\right)=B>0$.

Отсюда вытекает, что

$$
\begin{gathered}
\lim _{n \rightarrow \infty} \inf _{\substack{0 \leqslant Y_{1}-Y_{0} \leqslant h \\
Y_{1}, Y_{0} \in[0, b]}} \inf _{\mathbf{z} \in \mathscr{J}_{i}(k)} \mathbf{P}_{n}\left(\mathbf{Z}\left(m_{1} ; n\right)=\mathbf{z} \mid \mathbf{Z}\left(m_{0} ; n\right)=\mathbf{z},\left\|\mathbf{Z}\left(m_{2}, n\right)\right\| \leqslant k\right) \\
\geqslant(1-\chi h)^{N k} \frac{B}{B+\chi N k h} .
\end{gathered}
$$

Переходя к пределу при $h \downarrow 0$, получаем утверждение леммы 7 .

Следствие 1. Если выполнень условия леммы 7, то

$$
\begin{gathered}
\left\{\mathbf{Z}\left(\left(y+l_{n}\right) n^{1 / 2^{N-i}}, n\right), 0 \leqslant y \leqslant b \mid\left\|\mathbf{Z}\left(m_{2}, n\right)\right\| \leqslant k, \mathbf{Z}(n) \neq \mathbf{0}\right\} \\
\Longrightarrow\left\{\mathbf{U}_{i}(y), 0 \leqslant y \leqslant b \mid\left\|\mathbf{U}_{i}(2 b)\right\| \leqslant k\right\} .
\end{gathered}
$$

Д о к а з а т е л ь с т в о. Сходимость конечномерных распределений следует из соответствующих результатов о сходимости процессов, установленных в пункте 1) теоремы 2 работы [6]. Плотность вытекает из леммы 7 и теоремы 6 , если взять $\mathscr{B}=\mathscr{J}_{i}(k), \mathscr{C}=C_{i}(k)$ и заметить, что

$$
\begin{aligned}
\lim _{n \rightarrow \infty} & \mathbf{P}_{n}\left(\mathbf{Z}\left(l_{n} n^{\gamma_{i}}, n\right) \in \mathscr{C}_{i}(k) \mid\left\|\mathbf{Z}\left(m_{2}, n\right)\right\| \leqslant k\right) \\
& \leqslant \lim _{n \rightarrow \infty} \mathbf{P}_{n}\left(\underline{Z}_{i-1}\left(l_{n} n^{\gamma_{i}}\right)>0 \mid\left\|\mathbf{Z}\left(m_{2}, n\right)\right\| \leqslant k\right)=0 .
\end{aligned}
$$

Д ок аз а те ль с т о т е о ремы 2. Для $c>b$ положим

$$
\begin{aligned}
\mathbf{P}_{n, i}(b ;(\cdot)) & =\mathbf{P}_{n}\left(\left\{\mathbf{Z}\left(\left(y+l_{n}\right) n^{\gamma_{i}}, n\right), 0 \leqslant y \leqslant b\right\} \in(\cdot)\right), \\
\mathbf{P}_{n, i}^{(k)}(b, c ;(\cdot)) & =\mathbf{P}_{n}\left(\left\{\mathbf{Z}\left(\left(y+l_{n}\right) n^{\gamma_{i}}, n\right), 0 \leqslant y \leqslant b\right\} \in(\cdot)\|\| \mathbf{Z}\left(c n^{\gamma_{i}}, n\right) \| \leqslant k\right), \\
\overline{\mathbf{P}}_{n, i}^{(k)}(b, c ;(\cdot)) & =\mathbf{P}_{n}\left(\left\{\mathbf{Z}\left(\left(y+l_{n}\right) n^{\gamma_{i}}, n\right), 0 \leqslant y \leqslant b\right\} \in(\cdot)\|\| \mathbf{Z}\left(c n^{\gamma_{i}}, n\right) \|>k\right)
\end{aligned}
$$

И

$$
\begin{aligned}
\mathscr{P}_{i}(b ;(\cdot)) & =\mathbf{P}_{R_{i}}\left(\left\{\mathbf{U}_{i}(y), 0 \leqslant y \leqslant b\right\} \in(\cdot)\right), \\
\mathscr{P}_{i}^{(k)}(b, c ;(\cdot)) & =\mathbf{P}_{R_{i}}\left(\left\{\mathbf{U}_{i}(y), 0 \leqslant y \leqslant b\right\} \in(\cdot) \mid\left\|\mathbf{U}_{i}(c)\right\| \leqslant k\right) .
\end{aligned}
$$

Для $0<b<\infty$ и непрерывной действительной функции $\psi$ на $D_{[0, b]}\left(\mathbb{Z}_{+}^{N}\right)$ такой, что $|\psi| \leqslant q<\infty$, имеем

$$
\begin{aligned}
\int \psi(x) \mathbf{P}_{n, i}(b ; d x)= & \mathbf{P}_{n}\left(\left\|\mathbf{Z}\left(2 b n^{\gamma_{i}}, n\right)\right\|>k\right) \int \psi(x) \overline{\mathbf{P}}_{n, i}^{(k)}(b, 2 b ; d x) \\
& +\mathbf{P}_{n}\left(\left\|\mathbf{Z}\left(2 b n^{\gamma_{i}}, n\right)\right\| \leqslant k\right) \int \psi(x) \mathbf{P}_{n, i}^{(k)}(b, 2 b ; d x) .
\end{aligned}
$$


В силу свойств процесса $\mathbf{U}_{i}(\cdot)$ для первого слагаемого при $k \rightarrow \infty$ справедливы оценки

$$
\begin{aligned}
& \limsup _{n \rightarrow \infty} \mathbf{P}_{n}\left(\left\|\mathbf{Z}\left(2 b n^{\gamma_{i}}, n\right)\right\|>k\right) \int \psi(x) \overline{\mathbf{P}}_{n, i}^{(k)}(b, 2 b ; d x) \\
& \quad \leqslant q \limsup _{n \rightarrow \infty} \mathbf{P}_{n}\left(\left\|\mathbf{Z}\left(2 b n^{\gamma_{i}}, n\right)\right\|>k\right)=q \mathbf{P}_{R_{i}}\left(\left\|\mathbf{U}_{i}(2 b)\right\|>k\right)=o(1) .
\end{aligned}
$$

Далее, устремив к бесконечности сначала $n$, а затем $k$, получим

$$
\begin{aligned}
\lim _{k \rightarrow \infty} & \lim _{n \rightarrow \infty} \mathbf{P}_{n}\left(\left\|\mathbf{Z}\left(2 b n^{\gamma_{i}}, n\right)\right\| \leqslant k\right) \int \psi(x) \mathbf{P}_{n, i}^{(k)}(b, 2 b ; d x) \\
& =\lim _{k \rightarrow \infty} \mathbf{P}_{R_{i}}\left(0<\left\|\mathbf{U}_{i}(2 b)\right\| \leqslant k\right) \int \psi(x) \mathscr{P}_{i}^{(k)}(b, 2 b ; d x) \\
& =\lim _{k \rightarrow \infty} \int_{\left\{0<\left\|\mathbf{U}_{i}(2 b)\right\| \leqslant k\right\}} \psi(x) \mathscr{P}_{i}(b, 2 b ; d x)=\int \psi(x) \mathscr{P}_{i}(b ; d x) .
\end{aligned}
$$

Таким образом,

$$
\lim _{n \rightarrow \infty} \int \psi(x) \mathbf{P}_{n}\left(\mathbf{Z}\left(\left(\cdot+l_{n}\right) n^{\gamma_{i}}, n\right) \in d x\right)=\int \psi(x) \mathscr{P}_{i}(b ; d x)
$$

для любой непрерывной ограниченной функции $\psi$ на $D_{[0, b]}\left(\mathbb{Z}_{+}^{N}\right)$, что доказывает пункт 1) теоремы 2.

Доказательство пункта 2) теоремы 2 требует лишь несущественных изменений по сравнению с доказательством соответствующей теоремы работы [3], и мы его опускаем.

\section{4. Доказательство теорем 3 и 4.}

Д ок аз а т е л с с в о т е о р е м ы 3. Наши рассуждения основаны на следующем простом наблюдении:

$$
\left\{\bar{Z}_{1}(m, n)=1\right\} \Leftrightarrow\left\{\beta_{n} \geqslant m\right\} .
$$

Доказательство пункта 1). Согласно (15) при $m \ll n^{\gamma_{1}}$

$$
\begin{aligned}
\lim _{n \rightarrow \infty} \mathbf{P}_{n}\left(\bar{Z}_{1}(m, n)=1\right)= & \lim _{n \rightarrow \infty} \mathbf{P}_{n}\left(Z_{1}(m, n)=1\right) \\
& +\lim _{n \rightarrow \infty} \mathbf{P}_{n}\left(\bar{Z}_{2}(m, n)=1\right)=1+0=1 .
\end{aligned}
$$

Доказательство пункта 2). Заметим, что в силу (17)

$$
\begin{aligned}
& \lim _{n \rightarrow \infty} \mathbf{P}_{n}\left(\beta_{n} \geqslant y n^{\gamma_{i}}\right)=\lim _{n \rightarrow \infty} \mathbf{P}_{n}\left(\bar{Z}_{1}\left(y n^{\gamma_{i}}, n\right)=1\right) \\
& =\lim _{n \rightarrow \infty} \mathbf{P}_{n}\left(Z_{i}\left(y n^{\gamma_{i}}, n\right)+Z_{i+1}\left(y n^{\gamma_{i}}, n\right)=1\right) \\
& =\lim _{n \rightarrow \infty} \mathbf{P}_{n}\left(Z_{i}\left(y n^{\gamma_{i}}, n\right)=1 ; Z_{i+1}\left(y n^{\gamma_{i}}, n\right)=0\right) \\
& \quad+\lim _{n \rightarrow \infty} \mathbf{P}_{n}\left(Z_{i}\left(y_{n}^{\gamma_{i}}, n\right)=0 ; Z_{i+1}\left(y n^{\gamma_{i}}, n\right)=1\right) \\
& =-\left.\frac{\partial\left(\varphi_{i}\left(y ; s_{i}, s_{i+1}\right)\right)^{1 / 2^{i-1}}}{\partial s_{i}}\right|_{s_{i}=s_{i+1}=0}-\left.\frac{\partial\left(\varphi_{i}\left(y ; s_{i}, s_{i+1}\right)\right)^{1 / 2^{i-1}}}{\partial s_{i+1}}\right|_{s_{i}=s_{i+1}=0}
\end{aligned}
$$


Непосредственные вычисления показывают (см. (14)), что

$$
-\left.\frac{\partial \varphi_{i}\left(y ; s_{i}, s_{i+1}\right)}{\partial s_{i}}\right|_{s_{i}=s_{i+1}=0}=\frac{1-\tanh \left(y b_{i} c_{i, N}\right)}{1+\tanh \left(y b_{i} c_{i, N}\right)}=e^{-2 y b_{i} c_{i, N}}
$$

и

$$
-\left.\frac{\partial \varphi_{i}\left(y ; s_{i}, s_{i+1}\right)}{\partial s_{i+1}}\right|_{s_{i}=s_{i+1}=0}=\frac{\tanh \left(y b_{i} c_{i, N}\right)}{1+\tanh \left(y b_{i} c_{i, N}\right)}=\frac{1}{2}-\frac{1}{2} e^{-2 y b_{i} c_{i, N}} .
$$

Таким образом,

$$
\begin{aligned}
\lim _{n \rightarrow \infty} \mathbf{P}_{n}\left(Z_{i}\left(y n^{\gamma_{i}}, n\right)\right. & =1)=\frac{1}{2^{i-1}} e^{-2 y b_{i} c_{i, N}}, \\
\lim _{n \rightarrow \infty} \mathbf{P}_{n}\left(Z_{i+1}\left(y n^{\gamma_{i}}, n\right)=1\right) & =\frac{1}{2^{i}}\left(1-e^{-2 y b_{i} c_{i, N}}\right) .
\end{aligned}
$$

Комбинируя предыдущие оценки, получаем

$$
\lim _{n \rightarrow \infty} \mathbf{P}_{n}\left(\beta_{n} \leqslant y n^{\gamma_{i}}\right)=1-\frac{1}{2^{i}}-\frac{1}{2^{i}} e^{-2 y b_{i} c_{i, N}} .
$$

Доказательство пункта 3). Это утверждение очевидно.

Доказательство пункта 4). Необходимый результат следует из соотношения (18) и равенства

$$
-\left.\frac{\partial}{\partial s_{N}}\left(\frac{1}{x+(1-x) /\left(1-s_{N}\right)}\right)^{2^{-(N-1)}}\right|_{s_{N}=0}=\frac{1}{2^{N-1}}(1-x) .
$$

Д ок а з а т ль с т в о т е о р е м 4. Мы рассмотрим только случай $N \geqslant 4$ и $i \in\{2,3, \ldots, N-2\}$. Для $N=2,3$ или $N \geqslant 4$ и $i \in\{1, N-$ $1\}$ некоторые из появляющихся ниже случайных величин (событий) не существуют (пусты) и необходимые аргументы становятся короче.

Прежде всего заметим, что $\mathbf{P}_{n}\left(\beta_{n}<m\right)=\mathbf{P}_{n}\left(\mathbf{Z}_{1}(m, n) \geqslant 2\right)$, поскольку суммарное число частиц всех типов в редуцированном процессе не убывает от поколения к поколению. Положим $m_{i}=n^{\gamma_{i}\left(1+\gamma_{i}\right)}$, $i=1,2, \ldots, N-1$, и введем множество $\mathscr{H}_{i}=\left\{m: m_{i-1} \leqslant m \leqslant m_{i}\right\}$.

В силу (12)

$$
\begin{aligned}
& \mathbf{P}_{n}\left(\zeta_{n}=i ; \beta_{n}>m_{i}\right) \leqslant \mathbf{P}_{n}\left(\exists k>m_{i}: Z_{i}(k, n)>0\right) \\
& \leqslant \mathbf{P}_{n}\left(\exists k>m_{i}: Z_{i}(k)>0\right) \leqslant \mathbf{P}_{n}\left(Z_{i}\left(m_{i}\right)>0\right) \\
& \quad \leqslant \frac{\mathbf{P}\left(T_{i}>m_{i}\right)}{\mathbf{P}\left(T_{N}>n\right)}=o(1), \quad n \rightarrow \infty .
\end{aligned}
$$

Далее, по определению $\left\{Z_{i}(k, n)=1 ; \underline{Z}_{i-1}(k, n)+\bar{Z}_{i+1}(k, n)=0\right\}=$ $\left\{\mathbf{Z}(k, n)=\mathbf{e}_{i}\right\}$. Так как случайная последовательность $\bar{Z}_{j}(k, n), k \leqslant n$, 
не убывает по $k$ для каждого фиксированного $n$, а частицы типа $j$ могут производить потомков только типов, имеющих номера, не меньшие чем $j$, то

$$
\begin{aligned}
& \mathbf{P}_{n}\left(\zeta_{n}=i ; \beta_{n}<m_{i-1}\right)=\sum_{k=0}^{m_{i-1}-1} \mathbf{P}_{n}\left(\zeta_{n}=i ; \beta_{n}=k\right) \\
& \quad=\sum_{k=0}^{m_{i-1}-1} \mathbf{P}_{n}\left(Z_{i}(k, n)=1 ; \underline{Z}_{i-1}(k, n)+\bar{Z}_{i+1}(k, n)=0 ; \bar{Z}_{i}(k+1, n) \geqslant 2\right) \\
& \quad \leqslant m_{i-1} \max _{0 \leqslant k<m_{i-1}} \mathbf{P}_{n}\left(\mathbf{Z}(k, n)=\mathbf{e}_{i} ; \bar{Z}_{i}(k+1, n) \geqslant 2\right) \\
& \quad \leqslant m_{i-1} \max _{0 \leqslant k<m_{i-1}} \mathbf{P}_{n}\left(\bar{Z}_{i}(k+1, n) \geqslant 2 \mid \mathbf{Z}(k, n)=\mathbf{e}_{i}\right) .
\end{aligned}
$$

Ввиду (19) и равенства $H_{1}^{(i, N)}(\mathbf{s})=h_{i}(\mathbf{s})($ см. (1)) имеем

$$
\begin{aligned}
\mathbf{E}\left[\mathbf{s}^{\mathbf{Z}(k+1, n)} \mid \mathbf{Z}(k, n)=\mathbf{e}_{i}\right] & =1-\frac{1-H_{1}^{(i, N)}\left(\mathbf{1}-(\mathbf{1}-\mathbf{s}) \otimes \mathbf{Q}_{n-k-1}\right)}{Q_{n-k}^{(i, N)}} \\
& =1-\frac{1-h_{i}\left(\mathbf{1}-(\mathbf{1}-\mathbf{s}) \otimes \mathbf{Q}_{n-k-1}\right)}{Q_{n-k}^{(i, N)}}
\end{aligned}
$$

и, следовательно,

$$
\mathbf{E}\left[s_{i}^{Z_{i}(k+1, n)} \mid \mathbf{Z}(k, n)=\mathbf{e}_{i}\right]=1-\frac{1-h_{i}\left(1-\left(1-s_{i}\right) Q_{n-k-1}^{(i, N)} ; \mathbf{1}^{(N-i)}\right)}{Q_{n-k}^{(i, N)}}
$$

что в силу неравенства $\mathbf{P}_{n}\left(\bar{Z}_{i}(k+1, n)=1 \mid \mathbf{Z}(k, n)=\mathbf{e}_{i}\right) \geqslant \mathbf{P}_{n}\left(Z_{i}(k+\right.$ $\left.1, n)=1 \mid \mathbf{Z}(k, n)=\mathbf{e}_{i}\right)$ дает

$$
\begin{aligned}
& \mathbf{P}_{n}\left(\bar{Z}_{i}(k+1, n)=1 \mid \mathbf{Z}(k, n)=\mathbf{e}_{i}\right) \\
& \quad \geqslant\left.\frac{1}{Q_{n-k}^{(i, N)}} \frac{d h_{i}\left(1-\left(1-s_{i}\right) Q_{n-k-1}^{(i, N)} ; \mathbf{1}^{(N-i)}\right)}{d s_{i}}\right|_{s_{i}=0} \\
& \quad=\frac{Q_{n-k-1}^{(i, N)}}{Q_{n-k}^{(i, N)}} h_{i}^{\prime}\left(1-Q_{n-k-1}^{(i, N)} ; \mathbf{1}^{(N-i)}\right) \geqslant \frac{Q_{n-k-1}^{(i, N)}}{Q_{n-k}^{(i, N)}}\left(1-2 b_{i} Q_{n-k-1}^{(i, N)}\right) \\
& \geqslant 1-2 b_{i} Q_{n-k-1}^{(i, N)} \geqslant 1-2 b_{i} Q_{n-m_{i-1}}^{(i, N)} .
\end{aligned}
$$

Таким образом,

$$
\max _{0 \leqslant k<m_{i-1}} \mathbf{P}_{n}\left(\bar{Z}_{i}(k+1, n) \geqslant 2 \mid \mathbf{Z}(k, n)=\mathbf{e}_{i}\right) \leqslant 2 b_{i} Q_{n-m_{i-1}}^{(i, N)} .
$$

Отсюда, учитывая (12), выводим, что при $n \rightarrow \infty$

$$
\begin{aligned}
\mathbf{P}_{n}\left(\zeta_{n}=i ; \beta_{n}<m_{i-1}\right) & \leqslant 2 b_{i} m_{i-1} Q_{n-m_{i-1}}^{(i, N)} \\
& =O\left(n^{\gamma_{i-1}\left(1+\gamma_{i-1}\right)} n^{-\gamma_{i}}\right)=o(1) .
\end{aligned}
$$


Используя (26) и (27), заключаем, что при $n \rightarrow \infty$

$$
\begin{aligned}
\mathbf{P}_{n}\left(\zeta_{n}=i\right) & =\mathbf{P}_{n}\left(\zeta_{n}=i ; \beta_{n} \in \mathscr{H}_{i}\right)+o(1) \\
& =\mathbf{P}_{n}\left(\beta_{n} \in \mathscr{H}_{i}\right)-\mathbf{P}_{n}\left(\zeta_{n} \neq i ; \beta_{n} \in \mathscr{H}_{i}\right)+o(1) .
\end{aligned}
$$

Далее, в силу теоремы 1

$$
\mathbf{P}_{n}\left(\zeta_{n} \neq i ; \beta_{n} \in \mathscr{H}_{i}\right)=\mathbf{P}_{n}\left(\zeta_{n}=i+1 ; \beta_{n} \in \mathscr{H}_{i}\right)+o(1) .
$$

Применяя теперь (27) с заменой $i$ на $i+1$, мы видим, что при $n \rightarrow \infty$

$$
\begin{array}{r}
\mathbf{P}_{n}\left(\zeta_{n}=i+1 ; \beta_{n} \in \mathscr{H}_{i}\right) \leqslant \mathbf{P}_{n}\left(\zeta_{n}=i+1 ; \beta_{n} \leqslant m_{i}\right) \\
\leqslant 2 b_{i+1} m_{i} Q_{n-m_{i}}^{(i+1, N)}=O\left(n^{\gamma_{i}\left(1+\gamma_{i}\right)} n^{-\gamma_{i+1}}\right)=o(1) .
\end{array}
$$

Суммируя доказанные ранее оценки, получаем, что при $n \rightarrow \infty$

$$
\mathbf{P}_{n}\left(\zeta_{n}=i\right)=\mathbf{P}_{n}\left(\beta_{n} \in \mathscr{H}_{i}\right)+o(1) .
$$

Это с учетом (9) дает

$$
\lim _{n \rightarrow \infty} \mathbf{P}_{n}\left(\zeta_{n}=i\right)=\lim _{n \rightarrow \infty} \mathbf{P}_{n}\left(\beta_{n} \in \mathscr{H}_{i}\right)=\lim _{n \rightarrow \infty} \mathbf{P}_{n}\left(n^{\gamma_{i}} \ll \beta_{n} \ll n^{\gamma_{i+1}}\right)=\frac{1}{2^{i}},
$$

что и требовалось.

Наконец,

$$
\lim _{n \rightarrow \infty} \mathbf{P}_{n}\left(\zeta_{n}=N\right)=1-\sum_{i=1}^{N-1} \frac{1}{2^{i}}=\frac{1}{2^{N-1}} .
$$

Теорема 4 доказана.

\section{СПИСОК ЛИТЕРАТУРЫ}

1. Биллингсли П. Сходимость вероятностных мер. М.: Наука, 1977, 351 с.

2. Гихман И.И., Скороход А.В. Теория случайных процессов, т. 1. М.: Наука, 1971, $664 \mathrm{c}$.

3. Fleischmann K., Siegmund-Schultze R. The structure of reduced critical GaltonWatson processes. — Math. Nachr., 1977, v. 79, p. 233-241.

4. Foster J., Ney P. Decomposable critical multi-type branching processes. — Sankhyā A, 1976 , v. 38, № 1, p. 28-37.

5. Foster J., Ney P. Limit laws for decomposable critical branching processes. - Z. Wahrscheinlichkeitstheor. verw. Geb., 1978, v. 46, № 1, p. 13-43.

6. Ватутин B. A. Структура разложимых редуцированных ветвящихся процессов. I. Конечномерные распределения. - Теория вероятн. и ее примен., 2014, т. 59, в. 4, c. 667-692.

7. Ватутин B. А., Дьяконова E. E. Предельные теоремы для редуцированных ветвящихся процессов в случайной среде. — Теория вероятн. и ее примен., 2007, т. 52, в. 2, с. $271-300$. 